\title{
A Review on TSO-DSO Coordination Models and Solution Techniques
}

DOI:

10.1016/j.epsr.2020.106659

\section{Document Version}

Accepted author manuscript

Link to publication record in Manchester Research Explorer

\section{Citation for published version (APA):}

Givisiez, A. G., Petrou, K., \& Ochoa, L. F. (2020). A Review on TSO-DSO Coordination Models and Solution Techniques. Electric Power Systems Research, 189, 106659. https://doi.org/10.1016/j.epsr.2020.106659

\section{Published in:}

Electric Power Systems Research

\section{Citing this paper}

Please note that where the full-text provided on Manchester Research Explorer is the Author Accepted Manuscript or Proof version this may differ from the final Published version. If citing, it is advised that you check and use the publisher's definitive version.

\section{General rights}

Copyright and moral rights for the publications made accessible in the Research Explorer are retained by the authors and/or other copyright owners and it is a condition of accessing publications that users recognise and abide by the legal requirements associated with these rights.

\section{Takedown policy}

If you believe that this document breaches copyright please refer to the University of Manchester's Takedown Procedures [http://man.ac.uk/04Y6Bo] or contact uml.scholarlycommunications@manchester.ac.uk providing relevant details, so we can investigate your claim.

\section{OPEN ACCESS}




\title{
A Review on TSO-DSO Coordination Models and Solution Techniques
}

\author{
Arthur Gonçalves Givisiez ${ }^{1}$, Kyriacos Petrou ${ }^{1}$, Luis F. Ochoa ${ }^{1,2}$ \\ 1 The University of Melbourne, Melbourne, Australia \\ 2 The University of Manchester, Manchester, United Kingdom \\ arthurgivisiez@ieee.org; k.petrou@ieee.org; luis_ochoa@ieee.org
}

\begin{abstract}
The volume of services procured by transmission system operators (TSOs) through distribution-connected resources, aka distributed energy resources (DER), has been increasing in recent years. Currently, distribution networks are assumed to be fully capable of dealing with the resulting power flows. However, this assumption will no longer be valid as the volume of DER services become significant. Therefore, distribution system operators (DSOs) need to have a more active role to ensure the integrity of the distribution network while facilitating DER services. To achieve this, adequate coordination between TSOs and DSOs is required. To help stakeholders understand the implications of different coordination models so they can be adopted or tailored to their needs, this paper identifies three core TSO-DSO coordination models from the many proposed in the literature, discussing the corresponding advantages, disadvantages and challenges. Furthermore, a mapping of the proposed solution techniques is carried out to identify research trends and gaps.
\end{abstract}

Index Terms--Active distribution networks, Distributed Energy Resources (DER) Services, Distribution System Operator (DSO), TSO-DSO coordination models, TSO-DSO interaction.

\section{I.INTRODUCTION}

More and more, services procured by transmission system operators (TSOs) are being provided through distributed energy resources (DER), typically connected to medium (MV) and even low voltage (LV) distribution networks. Due to the relative scarcity of DER today, this procurement is done with the assumption that distribution networks are fully capable of dealing with the resulting time-varying power flows. In particular, given the rising adoption of photovoltaic (PV) and battery energy storage (BES) systems which offer significant controllability, the level of services that can be provided by consumers that also produce electricity (also known as prosumers) is rapidly increasing.

In distribution networks, large penetrations of DER alone can cause technical problems and challenges due to reverse power flows, such as network assets congestion [1] and voltage excursions [2, 3]. These issues, as demonstrated in [4] can be further exacerbated when controllable DER such as BES systems participate in the provision of services. In some coun- tries, like Australia, firm limits on DER exports are being imposed to help to deal with these issues. While this could be a viable intermediate solution that allows DER participating in the provision of services and satisfying network constraints, such traditional fit-and-forget approaches could also prove to be too prohibitive in the provision of services, effectively limiting the attractiveness of DER providing services through distribution networks.

As documented by reports released by organizations around the world in the last few years [1, 5-10], an alternative for countries/regions with open markets, is for distribution companies to have an active role in managing DER services (e.g., energy for balancing purposes, ancillary services, demand response, etc. - provided directly by prosumers or via aggregators) whilst also incorporating network constraints. This new role is commonly associated with the term Distribution System Operator (DSO) and has as objective the efficient facilitation of DER services by making the most of the existing infrastructure without compromising distribution network integrity [11-14]. The concept of having a DSO to manage DER services is similar to that of transactive energy [15].

Given that DSOs will inherently affect the way DER services will be provided, this new environment requires a paradigm shift in the way transmission and distribution interact. Consequently, having an adequate TSO-DSO coordination becomes crucial to ensure not only that the DSO role is fulfilled, but also the efficient operation of the power system.

To help stakeholders understand the implications of different coordination models so they can be adopted or tailored to their needs, this review paper identifies three core TSO-DSO coordination models from the many proposed in the literature, discussing the corresponding advantages, disadvantages and challenges.

In addition, solution techniques used to assess and solve network issues in each of the identified three TSO-DSO core models are mapped. These are classified regarding different types of optimization algorithms and their corresponding formulation. For completeness, information related to case studies are also presented, e.g., network information (e.g., MV,

This study was financed in part by the Coordenação de Aperfeiçoamento de Pessoal de Nível Superior - Brasil (CAPES) - Finance Code 001, and The University of Melbourne. 


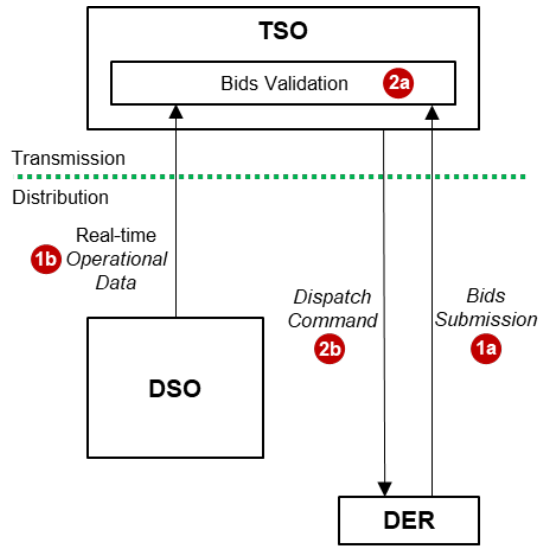

Figure. 1. TSO-managed model

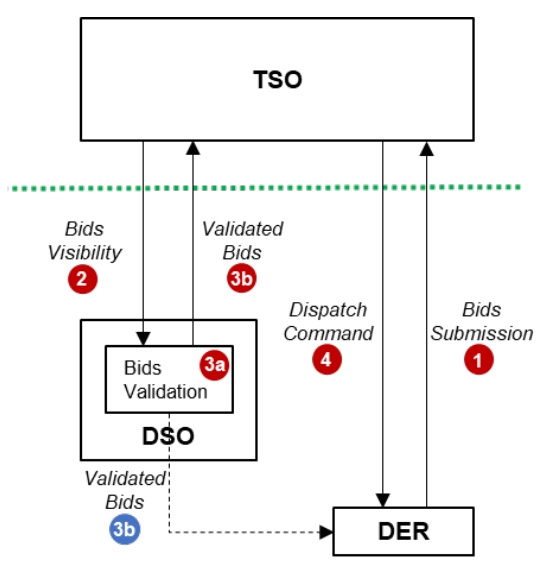

Figure. 2. TSO-DSO hybrid-managed model

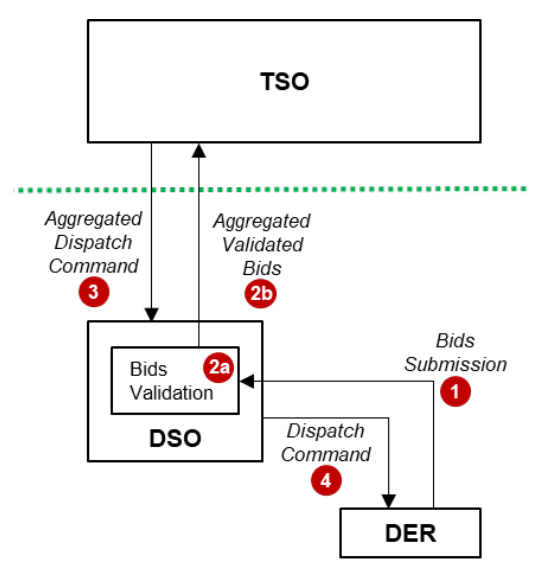

Figure. 3. DSO-managed model
$\mathrm{LV}$, transmission) and DER types and sizes. This mapping is important to identify promising techniques and their limitations, so they can be further investigated and improved.

This paper is structured as follows. In Section II, the TSODSO coordination is discussed, and the three identified models are presented and discussed in terms of their advantages, disadvantages and challenges. In Section III, the solution techniques used to assess and solve network issues in each of the identified models are mapped and discussed. Finally, the conclusions are presented in Section IV.

\section{II.TSO-DSO COORDINATION MODELS}

At the core of any TSO-DSO coordination model, in open market environments, is the consideration of distribution network constraints to facilitate and ensure the effective provision of DER services to the TSO. By catering for voltage and thermal limits (and any other potential limits, e.g., N-1 security, fault levels, etc.), it will be possible to validate services bids made by DER, i.e., to define which (or to what extent) services can be allowed without affecting the integrity of the distribution network at a given time. It is worth highlighting that in the mid-term, it is likely that all services will be ultimately needed and used by the TSO, i.e., there is only one central market for wholesale and ancillary services, as it is the case today around the world. However, in the future, certain TSO-DSO coordination models will also have to cater for distribution-level markets.

For clarity, it is important to highlight that the term "DER bids validation" is used here to refer to a process where DER bids (from prosumers and/or aggregators) are validated considering distribution network constraints. Depending on who carries out this task, this validation might be embedded in other processes. For instance, as it will be explained in the TSOmanaged model (Section II-A), the TSO could do this within its dispatch processes. Although the term "DER bids validation" is not usual, it allows clarifying this new process that will be required in all the TSO-DSO coordination models.

On one hand, the validation of DER services will allow a much higher certainty of the feasible volumes, which, in turn, allows the TSO to increase its reliance on DER, potentially reducing cost for the whole system. On the other hand, the adoption of any coordination model can increase both capital and operational expenditure of the distribution network. First, investments are required to increase network visibility and controllability, which at the initial stages will require significant investments. From a network asset perspective, costs are expected to increase due to quicker deterioration; result of the more effective utilization of the assets (i.e., lines and transformers operating closer to their physical limits for longer periods). Furthermore, the new active operation of the networks is likely to bring added operational costs.

There are also several socio-techno-economic challenges that need to be considered. The most prominent is the increased complexity of system operation and dispatch, due to a large number of DER and controllable network assets (e.g., on-load tap changers (OLTC), capacitor banks, etc.) to be orchestrated. Furthermore, adequate coordination relies on accurate network information, but as it currently stands, there is limited observability on distribution networks; especially on the LV level. The equitable use of the network is also an interesting challenge as determining the extent to which each DER can provide services should also consider some form of fairness. Increased access to network information makes cybersecurity even more important as now the system becomes more vulnerable to cyberattacks. Additionally, new policies and regulations need to be introduced to allow and facilitate for the transition towards TSO-DSO coordination models.

Although all TSO-DSO coordination model will share the aspects discussed above, the specifics of each model will bring advantages, disadvantages and challenges of their own. Nonetheless, most models available in the literature tend to be tailored to the needs of the individual power system/organization that proposed them; making it difficult to establish comparisons. Thus, the identification of core models (i.e., not specific to a given market structure/regulatory framework) is important as it enables researchers and industry alike to understand the advantages, disadvantages, and applicability of each model.

Accordingly, three core TSO-DSO coordination models are identified. In the first model, referred to as the TSOmanaged model, the TSO performs the economic dispatch of transmission energy resources (TER, conventional bulk gener- 
ation) and DER considering network constraints for both the distribution and transmission networks. In the second model, referred to as the TSO-DSO hybrid-managed model, the TSO performs the economic dispatch of TER and DER once the DSO has validated services bids made by DER to the central market. In the third model, referred to as the DSO-managed model, the DSO is responsible for validating, dispatching (in the presence of distribution-level markets) and aggregating all DER services. In the following subsections, these three models are presented and discussed in detail.

\section{A. TSO-Managed Model}

With this model, the TSO is responsible for the DER and TER dispatch [16-19] (i.e., a fully centralised dispatch) while accounting for both transmission and distribution networks constraints [20-22]. Depending on the energy market design, an independent market operator could also be the entity responsible for this task (e.g., Australian Energy Market Operator, AEMO, in Australia). Regardless of who performs the centralised dispatch, it must be clear that with this model one central entity [20-28] will be responsible for the whole system dispatch, and it has to ensure that both networks (i.e., transmission and distribution) will not have problems due to dispatch decisions.

The DSO's primary responsibilities (with regards to the coordination) is to provide distribution network real-time operational data to the TSO. Besides that, the DSO functions are limited to operating the distribution network, make investments and perform network maintenance [20]. Therefore, the TSO-DSO interaction is limited to the exchange of the realtime (or close to real-time) state of the distribution network participants (demand, generation, etc.) and assets (voltages, power flows, etc.).

Based on the above, the following sequence describes the core coordination process for a given service period (aka settlement or market clearance period). This is shown in Fig. 1.

1. DER send their bids directly to the TSO for a given service period (1a). The TSO also receives real-time operational data from the DSO (1b).

2. The TSO performs a centralised validation of DER services bids (2a) and computes the dispatch of TER and DER. The corresponding dispatch commands are sent to DER (2b).

In this model, the controllability of DER is technology independent. Since the DER bids are based on the prosumers' power injections (or absorptions), the TSO has only to model the injected/absorbed powers, i.e., the detailed modelling of the workings of DER by the TSO is not necessary. Of course, since the control of DER is done by an aggregator or the prosumer, it will be them doing the required detailed modelling. Finally, in this model, since all DER services are directed to the TSO, the simultaneous use of DER services by the TSO and DSO cannot occur.

1) Advantages: As a central entity, it is responsible for the whole system decision processes, simplifying the TSO-DSO coordination process. The adoption of new standards can be done in a faster and more straightforward way. Furthermore, as the TSO has know-how on dispatch processes, it can expand existing platforms. Finally, as the TSO is not the owner of the distribution assets, it is possible to make the most of it without any conflict of interest.

2) Disadvantages and Challenges: Since the DSO does not validate or dispatch DER, there is a risk of less efficient facilitation of services. In cases where the TSO is not able to control (directly or indirectly) DSO assets, there will be a less effective orchestration, reducing potential DER services. Validating and dispatching both the transmission and all distribution networks at once creates enormous computational and modelling challenges. Furthermore, as DER penetration increases, it becomes more difficult to coordinate the system in a fully centralised manner. Also, the volumes of real-time operational data to be transferred from the DSOs to the TSO can be significant. Finally, the TSO will have to interpret distribution network requirements, which is an area that the TSO does not have know-how. Table I summarizes the discussed points.

\section{B. TSO-DSO Hybrid-Managed Model}

In this model, seen in Fig. 2, the TSO is responsible for the DER and TER dispatch (i.e., a centralised dispatch), but it does not consider any distribution network aspect. The DSO is the entity responsible for validating DER services bids to guarantee network integrity. Accordingly, the process of validation starts with the visibility of the bids as proposed in [1, 5, $7,9,12,13,17,20,29-31]$. Once the bids are validated, this information can be sent to either the TSO or directly to DER, or even both at the same time. There is also the possibility of the DSO performing the validation before bids are sent to the TSO [28, 31-33], i.e., a pre-validation where DER send all bids first to the DSO who validates them so DER can directly send validated bids to the TSO.

Furthermore, the literature suggests that the DSO could also use DER services by procuring flexibility from the central market for distribution-related purposes such as mitigating distribution networks issues (operation) or even for investment deferral (planning) [5, 6, 8, 13, 17-19, 22, 29, 31].

Based on the aforementioned, the following sequence describes the core coordination process of this model.

1. DER send their bids directly to the TSO for a given service period.

2. The DSO is informed about the bids.

3. The DSO performs the validation of DER services bids (3a). Then, it transmits the validated bids and requests to the TSO (3b-red) and/or to DER (3b-blue).

4. The TSO performs the dispatch and sends the dispatch command to DER (4).

In this model, the controllability of DER is also technology independent for the TSO-DSO coordination, and it follows the same logic presented for the previous model. Furthermore, 
given that DER services are offered on the central market, both the TSO and DSO could decide to use it (if a specific market design allows it). Therefore, a priority has to be established (e.g., first the TSO, first the DSO, or based on the technical or economic criticality of the service). To establish this priority, it is important to also consider the type of the offered DER services, as it may be more useful to the DSO (e.g., services for local voltage problems or congestion) or to the TSO (e.g., energy balancing).

1) Advantages: As the DSO validates all DER bids, the know-how on network operation creates the opportunity to manage controllable assets in a way that facilitates more DER services compared to the TSO-managed model (and the TSO does not manage distribution network controllable assets). The computational and modelling requirements are reduced compared to the TSO-managed model as the assessment is restricted to the area of the DSO. The need for the DSO to transfer operational data to the TSO is also eliminated.

2) Disadvantages and Challenges: Since the DSO owns network assets and the facilitation of services requires using assets closer to their limits, the DSO might not push them as close to the limits as it could because this can reduce their lifetime. So, there is a possible conflict of interest for the DSO while managing network assets and validating DER services bids. Although the scale of the distribution networks managed by a single DSO cannot be compared with the whole power system (both transmission and all distribution networks together), there are still significant computational and modelling challenges. Furthermore, the coordination between TSO and DSO becomes more complex as the decision processes are not centralised in one entity.

\section{DSO-Managed Model}

This model can be interpreted as twofold, when there is no distribution-level market, and when such marketplace is present. When there is no distribution-level market, the TSO is responsible for TER and aggregated DER dispatch, however, the latter is done indirectly via the DSO. The DSO acts as the central coordinator of DER [1, 5, 7, 9, 14, 16, 17, 20, 21, 23$26,28,34-42]$, validating bids and providing them to the TSO as an aggregated volume of services [1, 7, 9, 20, 21, 41]. An aggregated dispatch command is sent by the TSO to the DSO who then dispatches individual DER to match the requirement.

Based on the above, the following sequence describes the core coordination process without the presence of distributionlevel markets, which is shown in Fig. 3.

1. DER send their bids to the DSO for a given service period.

2. The DSO performs validation of DER services bids and aggregates them (2a). Aggregated bids are submitted to the TSO (2b).
3. The TSO performs the dispatch and sends the aggregated dispatch command to the DSO.

4. The DSO sends the dispatch command to DER to match the aggregated requirement.

In this case, the controllability of DER depends on whether the DSO is the aggregator or not. If it is not, then it follows the same logic presented for the previous models. However, if the DSO is in fact the aggregator, then, the detailed modelling of DER needs to be done by the DSO in combination with the distribution network constraints. The complexity of DER modelling will depend on the technology (e.g., if only PV system is controlled, the modelling might not need to consider time aspects, but if there is a battery system, time aspects may be important). Furthermore, the core coordination process described before will start from a modified step 2, in which the DSO does not validate DER service bids but create/decide DER service bids and aggregate them (2a).

Furthermore, the transmission-distribution separation created by the DSO-managed model allows the creation of distribution-level markets where only DER participate. Two main schemes have been proposed in the literature:

i. The distribution-level market is cleared before DER bids are sent to the TSO market [5, 16-20, 43]. For this scheme, only steps 2 and 4 are modified. In step 2, the DSO performs the validation of DER services and makes the distribution-level market clearance. The remaining DER services bids are aggregated (2a). In step 4, both DSO and TSO send dispatch commands.

ii. The TSO sets requirements (or targets) at the TSODSO interface. The DSO is responsible for operating the distribution-level market (and corresponding DER bids) to achieve the TSO requirement whilst ensuring distribution network integrity [16-19, 42, 43]. For this scheme, only step 4 is modified. The DSO clears the distribution-level market and sends dispatch commands to DER to match the TSO's target.

Finally, in this model, the simultaneous use of DER services by the DSO and TSO is naturally solved when the DSO dispatches the distribution-level market before submitting the reminder bids to the TSO. However, in the case when the DSO has to follow the target set by the TSO, a priority process similar to the one discussed for the previous model should be considered.

1) Advantages: This model has the potential to achieve the highest level of efficiency on the orchestration of distribution network controllable assets and DER dispatch. If the TSO decides to request (dispatch) less than the available DER services, there is an opportunity for a re-optimization of the distribution network assets (e.g., reducing unnecessary control actions) because that information is processed by the DSO before sending the dispatch to DER. Similar to the previous model, the DSO leverages its know-how on distribution 
networks operation and there is no exchanges of distribution network operational data with the TSO.

2) Disadvantages and Challenges: A possible conflict of interest for the DSO when managing network assets and validating DER services bids also appears on this model. While the computational and modelling challenges of validating DER bids are similar to the previous model, the incorporation of distribution-level markets can add complexity which is aggravated by the lack of experience of the DSO on such processes (e.g., market design, market clearance, etc.).

The challenge of potential conflict of interest for the DSO, can be solved with an independent DSO (iDSO) [1, 7, 9, 20]. As the iDSO is a third-party entity, it can be neutral and transparent when managing network assets and DER. In this special case, the iDSO manages distribution network and/or markets, but it does not own network assets. In this case, there is still the existence of a traditional DSO which is responsible for making investments and maintaining the network in operational conditions, but also exchanges network status with the iDSO in real-time to allow the iDSO to operate the network.

However, independent entities would need to be created in each distribution network area. Consequently, more complexity would be added to the system operation, as the iDSO would have to exchange real-time network status with the DSO, and simultaneously, exchange market information with the TSO. Also, the cost of creating new entities in the system could undermine the benefits brought by iDSOs. Thus, an alternative to the iDSO model would be to keep the original DSO-managed model but imposing strong neutrality rules or providing regulation incentives to the DSO. The same can be applied to the TSO-DSO hybrid-managed model as it has similar challenges.

\section{III.SOLUTION TECHNIQUES}

As each of the identified TSO-DSO coordination models differs in the way that coordination is performed, solution techniques that apply to one might not necessarily apply to another, or at least has to be tailored accordingly. In order to accelerate research and innovation in the field, this section first identifies solution techniques and then maps them to each applicable model. A summary of solution techniques and simulation considerations for each model is presented in Table II

Although there are techniques based on rules using iterative power flows, the most common are based on optimization, in particular, using the optimal power flow (OPF). The latter are implemented using different optimization algorithms which can be classified as follows:

Distributed Optimization. An originally large-scale problem is decoupled in smaller problems (named subproblems, which are neighbours). For a given decoupled point, original variables (e.g., voltage, power) are duplicated, one for each neighbouring subproblem. This allows each subproblem to perform "local" decisions but maintaining some communication between neighbouring subproblems. In the end, neighbouring subproblems must match their decoupled variables, which in reality represents the same variable.

Hierarchical Optimization. An originally large-scale problem is divided in subproblems, which are organised in a hierarchical structure. Each subproblem performs local decisions and communicates them to the next hierarchical level.

Centralized Optimization. The large-scale problem is solved as a single problem.

Although the above optimization algorithms are applicable to all the TSO-DSO coordination models, the centralized optimization algorithm is commonly adopted by works investigating the TSO-managed model [22-28]. The corresponding problems were formulated using single-phase AC OPF for MV distribution networks [22-26, 28], while DC OPF was used for transmission networks [22-24, 28]. The exception is [27], which used AC OPF for transmission and data-driven aggregation of feasible regions for distribution networks.

In the TSO-DSO hybrid-managed model, the system dispatch is under the TSO responsibility and the validation of DER services bids is done by the DSO. In [28], validated bids are assumed to be given to the TSO, i.e., no actual validation process is considered. A centralised DC OPF is used to solve the dispatch problem. In [31, 32], only validation aspects are investigated, i.e., the TSO dispatch is not considered. An

TABLE I. TSO-DSO COORDINATION MODELS CHARACTERISTICS

\begin{tabular}{|c|c|c|c|}
\hline & TSO-Managed Model & TSO-DSO Hybrid-Managed Model & DSO-Managed Model \\
\hline Advantages & $\begin{array}{l}\text { - Simplifies the TSO-DSO coordination process } \\
\text { - TSO know-how on dispatch } \\
\text { - TSO has no conflict of interest when pushing } \\
\text { distribution network assets to the limits }\end{array}$ & $\begin{array}{l}\text { - Likely to have a more efficient facili- } \\
\text { tation of DER services } \\
\text { - DSO know-how on distribution net- } \\
\text { works } \\
\text { - Less computational and modelling } \\
\text { requirements (restricted to each DSO) } \\
\text { - No need for operational data transfer } \\
\text { from DSO to TSO }\end{array}$ & $\begin{array}{l}\text { - Likely to have the most efficient facili- } \\
\text { tation of DER services } \\
\text { - DSO know-how on distribution net- } \\
\text { works } \\
\text { - Less computational and modelling } \\
\text { requirements (restricted to each DSO) } \\
\text { - No need for operational data transfer } \\
\text { from DSO to TSO and less data ex- } \\
\text { changes between TSO and DSO/DER }\end{array}$ \\
\hline
\end{tabular}


iterative power flow is used to validate DER bids on MV networks. A complete process is proposed in [22] using a singlephase AC OPF for DER validation on distribution networks, while a DC OPF is used for system dispatch. In all cases, centralised optimization algorithms are used to solve the OPF.

Furthermore, in the DSO-managed model the system dispatch responsibility is divided between the TSO and the DSO, each one taking care of its own network. In [36, 38, 40, 44], the DSO optimizes DER services dispatch in order to send the dispatch command to DER (Fig. 3, step 4) and match the target given by the TSO. This is done using a single-phase AC OPF for MV networks and considering both centralized [36, 38, 40, 44] and distributed [36] optimization algorithms to solve the problem. In [35, 37-39, 44, 45], the validation (Fig. 3 , step 2a) and subsequent aggregation of DER services to be sent to the TSO (Fig. 3, step 2b) are performed by the DSO considering MV networks. In most cases, a centralised optimization algorithm is used to solve a single-phase AC OPF, but [39] proposes an iterative power flow approach. The complete process presented in Fig. 3 is captured by the techniques presented in [23-26, 28] using different optimization algorithms. In $[23,24,28]$, a distributed approach is used to decouple transmission network (DC OPF) from MV networks (singlephase AC OPF). In [25, 26] a hierarchical optimization algorithm is chosen to solve a single-phase AC OPF in three levels, which are represented by a simplified transmission network, a detailed MV network, and simplified LV networks.

From a scalability perspective, solution techniques need to ensure that TSOs and/or DSOs will be able to solve validation and dispatch problems on ultra large or large networks. However, most of the proposed techniques were tested in relatively small networks ( $\leq 900$ single-phase buses). So, even if some of the solution techniques show potential for scalability, it is not possible to affirm that such techniques are scalable. Other considerations include controllable network assets and the size of DER. Only a few papers consider extra flexibility brought by devices such as OLTCs on their solution techniques [22, 35, 37-40, 45]. Similarly, although the largest proportion of DER installations correspond to small and medium capacities $(<100 \mathrm{kVA})$, only a few papers investigate their validation and dispatch [27, 31, 32, 36, 44].

Based on the above, it is possible to identify trends and gaps on the literature of TSO-DSO coordination models. Most works adopt AC OPF formulations for distribution networks

TABLE II. LITERATURE MAPPING

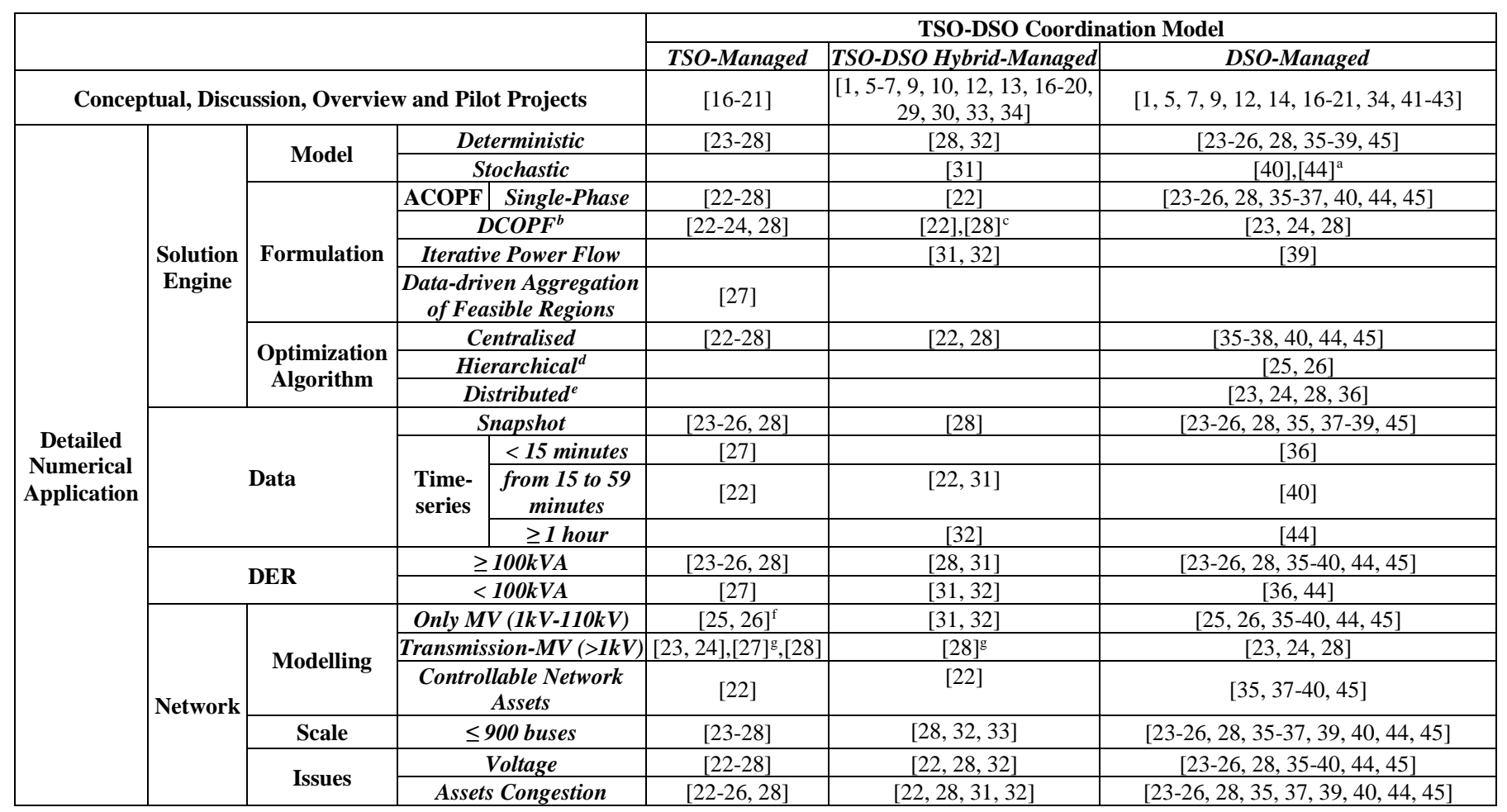

${ }^{\text {a }}$ If the aggregator is considered as the DSO it can be classified as DSO-Managed Model

b Transmission

${ }^{\mathrm{C}}$ It does not perform bids validation on distribution network, but it considers as the validation was performed before, so it makes the DCOPF for transmission

${ }^{\mathrm{d}}$ Between Transmission-MV-DER

${ }^{\mathrm{e}}$ Decoupled between Transmission-Distribution or between MV-DER

${ }^{\mathrm{f}}$ Really simplified transmission and LV network

${ }^{g}$ Only transmission network is considered 
but do not consider their unbalanced nature, (i.e., use singlephase formulations instead of three-phase). This is important for all types of distribution network design. For USA-style networks, MV feeders can be single, two or three-phase, whereas in the LV, only a few LV customers are directly connected to distribution transformers. For European-style networks, MV feeders are mostly three-phase, whereas distribution transformers supply multiple three-phase LV feeders and then dozens to hundreds of customers. Another aspect is that most works consider medium-scale DER and, therefore, model MV networks only. In reality, a large number of DER will be small (e.g., residential rooftop PV) and will be connected to lower voltages. Therefore, detailed LV networks and their interactions with MV networks will also need to be considered. It is important to highlight that only recently integrated MV-LV test feeders have been made available online (for instance, the USA-style IEEE 8500-Node Test Feeder made available in 2010 [46]). This scarcity of MV-LV test feeders might be part of the reason why only a few studies consider integrated MV-LV networks. Moreover, the consideration of controllable network assets (e.g., OLTCs), and their effects on DER validation, etc. is not well investigated. However, addressing all these aspects will add complexity to the validation and dispatch problem, bringing scalability challenges. Consequently, there will be a need for departing from centralized approaches and embrace the advantages of distributed optimization algorithms.

\section{IV.CONCLUSION}

The volume of services procured by transmission system operators (TSOs) through distributed energy resources (DER), has been increasing in recent years. Consequently, distribution system operators (DSOs) will soon be needed to have a more active role to ensure the integrity of the distribution network while facilitating DER services. To achieve this, adequate coordination between TSOs and DSOs is required. This paper first examined the relevant literature, using both academic and industry sources. Three core TSO-DSO coordination models were identified.

The first model, referred to as the TSO-managed model, can be considered to be the natural evolution of the model currently used around the world in which the TSO coordinates energy and service providers; with the added visibility of distribution networks and consideration of the corresponding technical constraints to validate DER services bids. However, as the volume of DER participating in markets and services increases in the future, this will become an ultra-large-scale problem significantly more difficult to solve.

The other two core models identified gives DSOs a more active role. In the TSO-DSO hybrid-managed model, the DER services bids validation is under the DSO responsibility, while the DER dispatch remains responsibility of the TSO. In the DSO-managed model, both the responsibility of DER services bids validation and DER dispatch falls on the DSO. These two models, however, increase the coordination complexity between the TSO and DSOs. They can also be affected by potential conflicts of interest from the DSO in regard to facilitating more DER services vs. the added cost that this will incur as their assets will be pushed to the limits. However, this could be addressed with adequate regulation (e.g., rules, incentives).

In summary, decision makers should evaluate the different core models and decide based on the characteristics that better fit the reality and needs of the corresponding power system. Certainly, the chosen core model will need to be tailored to the considered power system reality or its planned future.

Finally, this paper also mapped the solution techniques proposed in the literature for the different TSO-DSO coordination models. The single-phase AC OPF was the most common way of capturing the technical constraints and non-linearities of distribution networks when solving validation and dispatch problems of (mainly) medium-scale DER connected to MV levels. This, however, also highlights the need for comprehensive three-phase approaches that can not only handle the unbalance nature of distribution networks but also multiple voltages levels (e.g., MV and LV) so as to capture the effects of small-scale DER (e.g., residential rooftop PV) as well as controllable assets (e.g., OLTCs). This, in turn, will require exploring more the use of distributed optimization algorithms to solve such large-scale and complex problems.

\section{REFERENCES}

[1] AEMO and E. N. Australia, "Open Energy Networks Consultation Response," 2018.

[2] A. Navarro-Espinosa and L. F. Ochoa, "Probabilistic Impact Assessment of Low Carbon Technologies in LV Distribution Systems," IEEE Transactions on Power Systems, vol. 31, no. 3, pp. 2192-2203, 2016.

[3] A. T. Procopiou, K. Petrou, L. F. Ochoa, T. Langstaff, and J. Theunissen, "Adaptive Decentralized Control of Residential Storage in PV-Rich MV-LV Networks," IEEE Transactions on Power Systems, vol. 34, no. 3, pp. 2378-2389, 2019.

[4] K. Petrou, A. T. Procopiou, L. F. Ochoa, T. Langstaff, and J. Theunissen, "Impacts of Price-Led Operation of Residential Storage on Distribution Networks: An Australian Case Study," presented at the IEEE Milan PowerTech, Milan, Italy, 2019.

[5] E. N. Association, "Open Networks Project: Opening Markets for Network Flexibility," Energy Networks Association2017.

[6] Sweco, "Study on the Effective Integration of Distributed Energy Resources for Providing Flexibility to the Electricity System," 2015.

[7] AEMO and E. N. Australia, "Open Energy Networks Project: Workshop to Test Required Capabilities, Test Interactive Meta-Models and Discuss CBA Methodology," 2019.

[8] M. Miller et al., "Status Report on Power System Transformation: A 21st Century Power Partnership Report," National Renewable Energy Laboratory (NREL)2015.

[9] AEMO and E. N. Australia, "Open Energy Networks: Required Capabilities and Recommended Actions," 2019.

[10] CEDEC, ENTSO-E, GEODE, E.DSO, and EURELETRIC, "TSO-DSO Report: An Integrated Approach to Active System Management with the Focus on TSO-DSO Coordination in Congestion Management and Balancing," 2019.

[11] L. N. Ochoa, F. Pilo, A. Keane, P. Cuffe, and G. Pisano, "Embracing an Adaptable, Flexible Posture: Ensuring That Future European Distribution Networks Are Ready for More Active Roles," IEEE Power and Energy Magazine, vol. 14, no. 5, pp. 16-28, 2016.

[12] M. McGranaghan, D. Houseman, L. Schmitt, F. Cleveland, and E. Lambert, "Enabling the Integrated Grid: Leveraging Data to Integrate Distributed Resources and Customers," IEEE Power and Energy Magazine, vol. 14, no. 1, pp. 83-93, 2016. 
[13] S. Repo et al., "The IDE4L Project: Defining, Designing, and Demonstrating the Ideal Grid for All," IEEE Power and Energy Magazine, vol. 15, no. 3, pp. 41-51, 2017.

[14] P. Mallet, P.-O. Granstrom, P. Hallberg, G. Lorenz, and P. Mandatova, "Power to the People!: European Perspectives on the Future of Electric Distribution," IEEE Power and Energy Magazine, vol. 12, no. 2, pp. 51-64, 2014.

[15] D. Forfia, M. Knight, and R. Melton, "The View from the Top of the Mountain: Building a Community of Practice with the GridWise Transactive Energy Framework," IEEE Power and Energy Magazine, vol. 14, no. 3, pp. 25-33, 2016.

[16] G. Migliavacca et al., "SmartNet: H2020 project analysing TSO-DSO interaction to enable ancillary services provision from distribution networks," CIRED - Open Access Proceedings Journal, vol. 2017, no. 1, pp. 1998-2002, 2017.

[17] H. Gerard, E. I. Rivero Puente, and D. Six, "Coordination between transmission and distribution system operators in the electricity sector: A conceptual framework," Utilities Policy, vol. 50, pp. 40-48, 2018.

[18] C. Madina et al., "Cost-Benefit Analysis of TSO-DSO Coordination to Operate Flexibility Markets," presented at the 25th International Conference on Electricity Distribution, Madrid, Spain, 2019.

[19] L. Lind, R. Cossent, J. P. Chaves-Ávila, and T. Gómez San Román, "Transmission and distribution coordination in power systems with high shares of distributed energy resources providing balancing and congestion management services," Wiley Interdisciplinary Reviews: Energy and Environment, vol. 8, no. 6, 2019.

[20] P. D. Martini and L. Kristov, "Distribution Systems in a High Distributed Energy Resources Future: Planning, Market Design, Operation and Oversight," 2015.

[21] L. Kristov, "The Bottom-Up (R)Evolution of the Electric Power System: The Pathway to the Integrated-Decentralized System," IEEE Power and Energy Magazine, vol. 17, no. 2, pp. 42-49, 2019.

[22] M. Rossi et al., "Testing TSO-DSO Interaction Schemes for the Participation of Distributed Energy Resources in the Balancing Market: The Smartnet Simulator," presented at the 25th International Conference on Electricity Distribution, Madrid, Spain, 2019.

[23] H. Le Cadre, I. Mezghani, and A. Papavasiliou, "A game-theoretic analysis of transmission-distribution system operator coordination," European Journal of Operational Research, vol. 274, no. 1, pp. 317339, 2019.

[24] A. Mohammadi, M. Mehrtash, and A. Kargarian, "Diagonal Quadratic Approximation for Decentralized Collaborative TSO+DSO Optimal Power Flow," IEEE Transactions on Smart Grid, vol. 10, no. 3, pp. 2358-2370, 2019.

[25] Z. Yuan and M. R. Hesamzadeh, "Hierarchical coordination of TSODSO economic dispatch considering large-scale integration of distributed energy resources," Applied Energy, vol. 195, pp. 600-615, 2017.

[26] Z. Yuan, M. R. Hesamzadeh, and D. R. Biggar, "Distribution Locational Marginal Pricing by Convexified ACOPF and Hierarchical Dispatch," IEEE Transactions on Smart Grid, vol. 9, no. 4, pp. 31333142, 2018.

[27] E. Polymeneas and S. Meliopoulos, "Aggregate Modeling of Distribution Systems for Multi-Period OPF," presented at the Power Systems Computation Conference (PSCC), Genoa, Italy, 2016.

[28] A. Papavasiliou and I. Mezghani, "Coordination Schemes for the Integration of Transmission and Distribution System Operations," presented at the Power Systems Computation Conference (PSCC), Dublin, Ireland, 2018.

[29] A. Angioni et al., "A distributed automation architecture for distribution networks, from design to implementation," Sustainable Energy, Grids and Networks, vol. 15, pp. 3-13, 2018.

[30] U. S. D. o. Energy, "Modern Distribution Grid Decision Guide: Volume III," 2017.

[31] D. Koraki and K. Strunz, "Wind and Solar Power Integration in Electricity Markets and Distribution Networks Through Service-Centric Virtual Power Plants," IEEE Transactions on Power Systems, vol. 33, no. 1, pp. 473-485, 2018.
[32] J. A. P. Lopes, F. J. Soares, and P. M. R. Almeida, "Integration of Electric Vehicles in the Electric Power System," Proceedings of the IEEE, vol. 99, no. 1, pp. 168-183, 2011.

[33] EURELECTRIC, "Designing Fair and Equitable Market Rules for Demand Response Aggregation," 2015.

[34] S. Y. Hadush and L. Meeus, "DSO-TSO cooperation issues and solutions for distribution grid congestion management," Energy Policy, vol. 120, pp. 610-621, 2018.

[35] J. Silva et al., "Estimating the Active and Reactive Power Flexibility Area at the TSO-DSO Interface," IEEE Transactions on Power Systems, vol. 33, no. 5, pp. 4741-4750, 2018.

[36] E. Dall'Anese, S. S. Guggilam, A. Simonetto, Y. C. Chen, and S. V. Dhople, "Optimal Regulation of Virtual Power Plants," IEEE Transactions on Power Systems, vol. 33, no. 2, pp. 1868-1881, 2018.

[37] J. Silva, J. Sumaili, R. J. Bessa, L. Seca, M. Matos, and V. Miranda, "The challenges of estimating the impact of distributed energy resources flexibility on the TSO/DSO boundary node operating points," Computers \& Operations Research, vol. 96, pp. 294-304, 2018.

[38] N. Fonseca et al., "EvolvDSO Grid Management Tools to Support TSO-DSO Cooperation," presented at the CIRED Workshop, Helsinki, Finland, 2016.

[39] M. Heleno, R. Soares, J. Sumaili, R. J. Bessa, L. Seca, and M. A. Matos, "Estimation of the Flexibility Range in the TransmissionDistribution Boundary," presented at the IEEE Eindhoven PowerTech, Eindhoven, Netherlands, 2015.

[40] A. Saint-Pierre and P. Mancarella, "Active Distribution System Management: A Dual-Horizon Scheduling Framework for DSO/TSO Interface Under Uncertainty," IEEE Transactions on Smart Grid, vol. 8, no. 5, pp. 2186-2197, 2017.

[41] J. Villar et al., "Flexibility Hub - Multi Service Framework for Coordination of Decentralised Flexibilities," presented at the 25th International Conference on Electricity Distribution, Madrid, Spain, 2019.

[42] M. Pardo, C. Madina, M. Marroquín, and E. Estrade, "Use of Radio Base Stations to Provide Ancillary Services to the DSO Through Local Flexibility Markets," presented at the 25th International Conference on Electricity Distribution, Madrid, Spain, 2019.

[43] Y. Tohidi, M. Farrokhseresht, and M. Gibescu, "A Review on Coordination Schemes Between Local and Central Electricity Markets," presented at the 15th International Conference on the European Energy Market (EEM), Lodz, Poland, 2018.

[44] B. Vatandoust, A. Ahmadian, M. A. Golkar, A. Elkamel, A. Almansoori, and M. Ghaljehei, "Risk-Averse Optimal Bidding of Electric Vehicles and Energy Storage Aggregator in Day-Ahead Frequency Regulation Market," IEEE Transactions on Power Systems, vol. 34, no. 3, pp. 2036-2047, 2019.

[45] F. Capitanescu, "TSO-DSO interaction: Active distribution network power chart for TSO ancillary services provision," Electric Power Systems Research, vol. 163, pp. 226-230, 2018.

[46] R. C. Dugan and R. F. Arritt, "The IEEE 8500-Node Test Feeder," presented at the IEEE PES T\&D 2010, New Orleans, LA, USA, 2010. 